\title{
ZU DEN MIT ÄGYPTISCH 'IRP, „WEIN“ GEBILDETEN WORTSPIELEN
}

\author{
STEFAN BOJOWALD
}

\begin{abstract}
Ägyptologisches Seminar der Universität Bonn Regina-Pacis-Weg 7, 53113 Bonn, Deutschland e-mail: stefan.bojowald@t-online.de
\end{abstract}

This paper gives an overview of plays on words with the Egyptian irp 'wine'. In total, seven examples could be found, which are introduced individually and, as much as possible, reduced to origins related to sounds. The relatively large number of plays on words built with irp 'wine' can be explained by the importance of wine in cultural history.

Key words: Egyptian philology, Egyptian etymology, Egyptian word irp 'wine'.

Die ägyptische Sprache blickt bekanntlich auf ein reiches Erbe an Wortspielen zurück. Der Vergleich mit den orientalischen Nachbarsprachen läuft z. T. auf ganz ähnliche Resultate hinaus. Was die ägyptischen Wortspiele anbelangt, so sind sie durch die gesamte Sprachgeschichte hindurch zu beobachten. Die Wortspiele treten darüber hinaus unabhängig von der jeweiligen Textgattung auf. Die Haupttypen der ägyptischen Wortspiele sind bereits von Guglielmi (1986) überblickartig zusammengefasst worden. Die Sprachwissenschaft pflegt generell zwischen impliziten (immanenten)/vertikalen und expliziten/ horizontalen Wortspielen zu differenzieren. Das implizite (immanente)/vertikale Wortspiel basiert auf der Doppeldeutigkeit eines Ausdrucks (Amphibolie), während beim expliziten/ horizontalen Wortspiel die Identität des Ausdrucks den Eindruck einer Kontinuität des Ausdrucks auf inhaltlicher Ebene erweckt. Die literarischen Funktionen der Wortspiele können in textkonstituierende Elemente, Textklammern (Inclusiones) oder Euphemismen unterteilt werden (Guglielmi 1984, pp. 491-506).

In diesem Beitrag sollen die Wortspiele mit irp „Wein“ (Calice 1936, p. 120; Faulkner 1962, p. 28; Meeks 1980, p. 39; 1981, p. 43; 1982, pp. 30-31; Lesko 1982, p. 47; Wilson 1997, pp. 95-96; Hannig 2003, pp. 191-198; 2006, pp. 377-380) näher betrachtet werden. Die folgenden allgemeinen Angaben seien der Untersuchung als Einführung vorausgeschickt. 
Das Wort ist bis in die jüngsten Sprachstufen hinein gebraucht worden. Die alte Wortgestalt hat noch im Demotischen Bestand gehabt, wo es als irp (Erichsen 1954, p. 39) erscheint. Im Koptischen ist die Form „нрп“ (Westendorf 1965/1977, p. 46; Černý 1976, p. 42; Vycichl 1983, pp. 54-55) verwendet worden. Die Mitteilung von einigen Sonderschreibungen des Wortes dürfte ebenfalls nicht ganz unnütz sein. Die erste Erwähnung hat darunter die Defektivschreibung ip (Kitchen 1975, Vol. I, p. 59, line 12; Roquet 1977, p. 114, note 1) verdient, bei der $r$ an $i$ assimiliert ist. Die Voraussetzung hierfür bildet der Lautwandel zwischen $i$ und $r$, der auch sonst recht häufig durchgeführt worden ist (Westendorf 1962, p. 32). Das gleiche Interesse ruft die Schreibung ir (Hassan 1944, pp. 94, 118; Quibell 1923, Plate XXVI; Balcz 1932, p. 63) hervor, bei der es zum Ausfall von $p$ gekommen ist. Die Beispiele für den Ausfall von $p$ sollen bei einer anderen Gelegenheit besprochen werden. Die Schreibung irwp (Jansen-Winkeln 2007, p. 167) verlangt dieselbe Aufmerksamkeit, bei der ein $w$ in die Schreibung gelangt ist. Der Zusatz eines bedeutungslosen $w$ im Inlaut ist durch Westendorf (Westendorf 1962, p. 21) an mehreren Beispielen aufgezeigt worden. Das ägyptische Wort irp „Wein“ ist möglicherweise in die ugaritische Sprache entlehnt worden (Muchiki 1999, p. 281). Der gleiche Entlehnungsprozess hat in die nubische Sprache stattgefunden (Khalil 1988, p. 79).

Der Schwerpunkt dieser Arbeit wird folglich auf den mit irp „Wein“ gebildeten Wortspielen liegen, deren zusammenfassende Behandlung bisher noch ausgestanden hatte. Das Thema des Artikels darf daher mit einiger Berechtigung als neu bezeichnet werden. Die Grundlage der folgenden Ausführungen wird aus insgesamt sieben verschiedenen Wortspielen bestehen. Die Möglichkeit, dass Beispiele schlichtweg übersehen worden sind, kann zwar nie mit hundertprozentiger Sicherheit ausgeschlossen werden. In Zukunft könnten daher noch Belege hinzukommen, so dass die Zahl u. U. nach oben korrigiert werden muss. Die bisherigen Beispiele stellen aber für ein einzelnes Nomen eine recht große Menge dar. Die Basis reicht daher aus, um erste, vorsichtige Rückschlüsse zu erlauben.

Das erste Wortspiel kann zwischen irp „Wein“ und 'Imr „Amor“ beobachtet werden, das sich in irp $n$ 'Imr (Gardiner 1947a, p. 187*; 1947b, p. 236*) (Neues Reich) „Wein von Amor“ entwickelt hat. Das Wortspiel hat sich auf den Lautwandel zwischen $p$ und $m$ gestützt, der unter bestimmten Voraussetzungen von Vittmann (Vittmann 1998, p. 554, note 1547) behandelt worden ist. Wenn diese Erklärung zu schwach begründet ist, könnte der Lautwandel zwischen $p$ und $m$ theoretisch auch als Analogiebildung zum Lautwandel zwischen $b$ und $m$ betrachtet werden können, der sehr viel besser bezeugt ist (Westendorf 1962, p. 23). Der Lautwandel zwischen $b$ und $p$ (Westendorf 1962, p. 23) würde sich als Bindeglied empfehlen.

Das zweite Wortspiel zwischen irp „Wein“ und irp „berauschen“ tritt als nächstes hinzu, das sich in: hnw.t 3h.t hw.t - hr, nb(.t) [3h.]w [3h.t r 3h.w, irp.t $r$ irp.w (Sternberg el Hotabi 1992, p. 21) (Spätzeit) feststellen lässt. Die Übersetzung mit: „strahlende Fürstin, Hathor, Herrin der Strahlenden, [die die Strahlenden überstrahlt, und berauschender ist als Wein" dürfte den Ansprüchen am besten genügen. Das Wortspiel ist besonders leicht zu identifizieren, weil es sich bei irp „Wein“ und irp 
„berauschen“ um zwei homonyme Wurzeln handelt. Die Möglichkeit ist sogar nicht ausgeschlossen, dass die Wurzeln etymologisch zusammenhängen.

Das dritte Wortspiel ist zwischen irp „Wein“ und 'pr ,ausstatten“ zur Ausführung gelangt, das in irp.w wr.w (n.)w knm.t dśdś 'pr $m$ ir.t - hr w3 $\underline{d}$ (Waitkus, 1997, p. 151) (Spätzeit) zu finden ist. Die Stelle kann am sinnvollsten mit „die großen Weine aus Charga und Bahrija, die ausgestattet ${ }^{1}$ sind als grünes Horusauge“ übersetzt werden. Die Ursache für das Wortspiel hat der Lautwandel zwischen ' und $i$ gebildet, der zu den häufigsten derartigen Phänomenen in der ägyptischen Sprache überhaupt gerechnet werden muss (vgl. Sethe 1899, pp. 88, 90; Peust 1999, pp. 103-104; Westendorf 1962, p. 19).

Das vierte Wortspiel ist zwischen irp „Wein“ und wpi r $r$,Mund öffnen“ vonstatten gegangen, das in ' irp 2, 'Imn $m n n=k i r . t-h r$ wpi $r$ ' $=k$ im $={ }^{\prime}$ ' (Tacke 2013b, p. 104) (Neues Reich) zu belegen ist. Die Wiedergabe mit: ' - Näpfe von Wein: 2 Stück: Amun, nimm dir das Auge des Horus, möge dein Mund damit geöffnet werden." lässt sich hierfür wohl am besten vertreten. Dass hier ein Wortspiel gegeben ist, hat bereits Tacke erkannt. Im Prinzip stehen bei der Erklärung des Wortspiels zwei Wege offen. Im ersten Fall könnte mit dem Lautwandel zwischen $i$ und 3 bei gleichzeitigem Verlust von $w$ zu Beginn des Wortes operiert werden. Der hierfür notwendige Lautwandel zwischen $i$ und 3 kommt in der ägyptischen Sprache fast schon inflationär häufig vor (Sethe 1899, p. 48; Edel 1955/1964, pp. 59f; Westendorf 1962, pp. 10f; JasnowZauzich 2005, p. 89; Jansen-Winkeln 1996, p. 32 §47). Der Ausfall von $w$ zu Beginn des Wortes ist in der Literatur ebenfalls schon öfter behandelt worden (Westendorf 1962, p. 20; Kurth 2007, p. 493). Im zweiten Fall könnte mit dem Lautwandel zwischen $w$ und $i$ bei gleichzeitigem Ausfall von 3 am Ende des Wortes argumentiert werden. Der Lautwandel zwischen $w$ und $i$ hat sich in der ägyptischen Sprache weiter Verbreitung erfreut (Sethe 1899, pp. 87f, 116f; Edel 1955/1964, p. 62). Der Ausfall von 3 am Ende des Wortes darf aufgrund der Untersuchungen von Sethe (1899, pp. 42ff) und Westendorf (1962, p. 9) als fest etabliert gelten. Das Wortspiel kommt noch an zwei weiteren Stellen ${ }^{2}$ vor, was auf eine gewisse Beliebtheit schließen lässt. Dass alle Wortspiele im gleichen Texttyp begegnen, braucht der Einschätzung nicht zu widersprechen. Der zweite Nachweis des Wortspiels ist in: irp, ' 2, 'Imn $m n n=k$ ir.thr wpi $r 3=k i m=\hat{s}$ (Tacke 2013b, p. 212) (Neues Reich) aufzudecken, wofür die Übersetzung ,irp - Wein, zwei Näpfe: Amun, nimm dir das Auge des Horus, möge dein Mund damit geöffnet werden." die beste Alternative darstellt. Der dritte Beleg für das Wortspiel liegt in: hnk $m$ irp, 'Imn $m n=k[i r . t]-h r$ wpi $r\}=k i m=s$ (Tacke 2013b, p. 242) (Neues Reich) vor, wofür die Übersetzung „Beschenken mit Wein: Amun, nimm dir [das Auge] des Horus; möge dein Mund damit geöffnet werden." am weitesten führen dürfte.

Das fünfte Wortspiel kann zwischen irp „Wein“ und $\mathrm{Pr}-{ }^{3}$ „,König“ angenommen werden, das in irp Pr - 3 (Erichsen 1954, p. 39) (Spätzeit) „Wein des Königs“ zu beobachten ist. Die Hauptbedingung für das Wortspiel ist entweder im Lautwandel

\footnotetext{
${ }^{1}$ Die von Waitkus für ` $p r$ gewählte Bedeutung „darbringen“ entspricht nicht den üblichen Konventionen und sollte besser in der praktizierten Weise ersetzt werden.

${ }^{2} \mathrm{Zu}$ einem möglichen weiteren Beispiel vgl. Tacke (2013a, p. 109).
} 
zwischen ' und $i$ oder 3 und $i$ zu suchen. Das Wortspiel wird zusätzlich durch eine Metathese abgerundet.

Das sechste Wortspiel kann zwische irp „Wein“ und pri „herauskommen“ konstatiert werden, das in $m n n=k$ irp pri $m$ dśdś, ir.t hr pri $m$ knm.t.t (Sethe-Firchow 1957, Vol. VIII, pp. 4, 9) (Spätzeit) stattgefunden hat. Die Übersetzung „Empfange den Wein, der aus Bahrija kommt, das Horus - Auge, das aus Kenset kommt" (Sternberg el Hotabi 1993, p. 34) dürfte am meisten Wahrscheinlichkeit besitzen. Die Entstehung des Wortspieles ist über eine Metathese gelaufen.

Das siebte Wortspiel setzt sich aus den Komponenten irp „Wein“ und rnpi „,verjüngen“ zusammen, das in $\check{s} p s^{\prime}=k m \check{s}$ 3, rnpi=k $m$ irp (Chassinat 1928, p. 176; Poo 1984 , p. 128; 1995, p. 126) (Spätzeit) nachzuweisen ist. Die Wiedergabe „mögest du verherrlicht werden durch $\breve{s} 3$ - Wein, mögest du verjüngt werden durch irp - Wein“ dürfte der Realität am nächsten kommen. In der Erklärung des Wortspiels kann zwischen zwei Alternativen gewählt werden. Die erste Möglichkeit liegt im Ausfall von $n$, der bereits mehrfach erörtert worden ist (Westendorf 1962, p. 27; JansenWinkeln 1996, p. 36). Die zweite Möglichkeit bildet der Zusatz von $n$, über den Westendorf geschrieben hat (Westendorf 1962, p. 29).

Die abschließende Bewertung könnte etwa wie folgt aussehen. Die Hochschätzung gerade des Weines in Wortspielen, von der schon beim jetzigen Stand auszugehen ist, kann vielleicht mit der kulturgeschichtlichen Bedeutung des Getränkes begründet werden. Der Anbau und die Pflege der Weinrebe sind bekanntlich unter die zivilisatorischen Meisterleistungen einzureihen. Die Wortspiele haben der wichtigen Funktion des Weines in Leben und Alltag der damaligen Menschen ein sprachliches Denkmal gesetzt ${ }^{3}$.

\section{Bibliographie}

Balcz, H. (1932): Die Gefässdarstellungen des Alten Reiches. Mitteilungen des Deutschen Archäologischen Instituts Kairo Vol. 3, pp. 50-87.

Calice, Fr. (1936): Grundlagen der ägyptisch-semitischen Wortvergleichung. Eine kritische Diskussion des bisherigen Vergleichsmaterials. In: Beihefte zur Wiener Zeitschrift für die Kunde des Morgenlandes, 1. Heft. Wien.

Černý, J. (1976): Coptic Etymological Dictionary. Cambridge-London-New York-Melbourne.

Chassinat, (1928): Le temple d'Edfou. Tome Troisième. Le Caire.

Edel, E. (1955/1964): Altägyptische Grammatik. Rom (Analecta Orientalia 34/39).

Erichsen, W. (1954): Demotisches Glossar. Kopenhagen.

Faulkner, R. O. (1962): A Concise Dictionary of Middle Egyptian. Oxford.

Gardiner, A. H. (1947a): Ancient Egyptian Onomastica. Text, Volume I. Oxford.

Gardiner, A. H. (1947b): Ancient Egyptian Onomastica. Text, Volume II. Oxford.

Grapow, H. (1924): Die bildlichen Ausdrücke des Aegyptischen. Vom Denken und Dichten einer altorientalischen Sprache. Leipzig. 173).

${ }^{3} \mathrm{Zu}$ Vergleichen mit Wein (auch mit anderen Weinbezeichnungen) vgl. Grapow (1924, p. 
Guglielmi, W. (1984): Zu einigen literarischen Funktionen des Wortspiels. In: Studien zur Sprache und Religion Ägyptens. Band 1, Festschrift für W. Westendorf. Göttingen, pp. 491-506.

Guglielmi, W. (1986): Wortspiel. In: Helck, W. - Westendorf, W. (Hrsg.): Lexikon der Ägyptologie, Band VI. Wiesbaden, Stele-Zypresse, pp. 1287-1291.

Hannig, R. (2003): Ägyptisches Wörterbuch I. Altes Reich und Erste Zwischenzeit. Mainz.

Hannig, R. (2006): Ägyptisches Wörterbuch II. Mittleres Reich und Zweite Zwischenzeit. Teil 1. Mainz.

Hassan, S. (1944): Excavations at Gîza, with Special Chapters on Methods of Excavation, the False Door, and Other Archaeological and Religious Subjects. Vol. V. 1933-1934. Excavations of the Faculty of Arts, Fouad I University, Service des Antiquitès de l'Égypte. Cairo.

Jansen-Winkeln, K. (1996): Spätmittelägyptische Grammatik der Texte der 3. Zwischenzeit. Wiesbaden (Ägypten und Altes Testament 34).

Jansen-Winkeln, K. (2007): Inschriften der Spätzeit. Teil 1: Die 21. Dynastie. Wiesbaden.

Jasnow, R. - Zauzich, K.-Th. (2005): The Ancient Egyptian Book of Thot, A Demotic Discourse on Knowledge and Pendant to the Classical Hermetica. Volume 1: Text. Wiesbaden.

Khalil, M. (1988): Studien zum Altnubischen. Nubisch-ägyptische Beziehungen. Frankfurt-BernNew York-Paris (Europäische Hochschulschriften Reihe XXVII, Asiatische und Afrikanische Studien Band 19).

Kitchen, Kenneth A. (1975): Ramesside Inscriptions, Historical and Biographical I. Oxford.

Kurth, D. (2007): Einführung ins Ptolemäische. Eine Grammatik mit Zeichenliste und Übungsstücken. Teil 1. Hützel.

Lesko, L. H. (1982): A Dictionary of Late Egyptian. Volume I. Berkeley.

Meeks, D. (1980): Annee lexicographique. Tome I (1977). Paris.

Meeks, D. (1981): Annee lexicographique, Egypte Ancienne. Tome 2 (1978). Paris.

Meeks, D. (1982): Annee lexicographique, Egypte Ancienne. Tome 3 (1979). Paris.

Muchiki, Y. (1999): Egyptian Proper Names and Loanwords in North-West Semitic. Atlanta, Society of Biblical Literature (Dissertation Series Number 173).

Peust, C. (1999): Egyptian Phonology. An Introduction to the Phonology of a Dead Language. Göttingen.

Poo, M.-Ch. (1984): The Offering of Wine in Ancient Egypt. Baltimore.

Poo, M.-Ch. (1995): Wine and Wine Offering in the Religion of Ancient Egypt. London-New York (Studies in Egyptology 22).

Quibell, J. E. (1923): Excavations at Saqqara (1912-1914), Archaic Mastabas. Service des Antiquitès de l'Égypte. Le Caire.

Roquet, G. (1977). Le nom du "Lunx" I $(n) b$ comme Anthroponyme ( $3^{\mathrm{e}}$ Dynastie). Bulletin de l'Institut Français de l'Archéologie Orientale Vol. 77, pp. 113-117.

Sethe, K. (1899). Das aegyptische Verbum im Altaegyptischen, Neuaegyptischen und Koptischen. Erster Band, Laut- und Stammes-lehre. Leipzig.

Sethe, K.-Firchow, O. (1957): Thebanische Tempelinschriften aus griechisch-römischer Zeit, Band I. Berlin.

Sternberg el Hotabi, H. (1992): Ein Hymnus an die Göttin Hathor und das Ritual „Hathor das Trankopfer darbringen“ - nach den Tempeltexten der griechisch-römischen Zeit, unter Mitarbeit von Frank Kammerzell. Teil I: Kammerzell, Frank-Sternberg el Hotabi, H.: Quelle und Bearbeitung der Hauptzeugen. Teil II: Sternberg el Hotabi, H.: Weitere Textzeugen, Gesamtinterpretation und Indices. Bruxelles (Rites Éyptiens VII).

Sternberg el Hotabi, H. (1993): Der Propylon des Month-Tempels in Karnak-Nord. Zum Dekorationsprinzip des Tores, Übersetzung und Kommentierung der Urkunden, VIII, Texte Nr. 1 Nr. 50. Wiesbaden (Göttinger Orientforschungen IV Reihe: Ägypten, Band 25). 
Tacke, N. (2013a): Das Opferritual des ägyptischen Neuen Reiches. Band I: Texte. Leuven-ParisWalpole, Massachusetts (Orientalia Lovaniensia Analecta 222).

Tacke, N. (2013b): Das Opferritual des ägyptischen Neuen Reiches. Band II: Übersetzung und Kommentar. Leuven-Paris-Walpole, Massachusetts (Orientalia Lovaniensia Analecta 222).

Vandier, J. (1961): Le Papyrus Jumilhac. Paris.

Vittmann, G. (1998): Der demotische Papyrus Rylands 9. Teil II, Kommentare und Indizes. Wiesbaden (Ägypten und Altes Testament 38).

Vycichl, W. (1983): Dictionnaire Étymologique de la langue Copte. Leuven.

Waitkus, W. (1997): Die Texte in den unteren Krypten des Hathortempels von Dendera. Ihre Aussage zur Funktion und Bedeutung dieser Räume. Mainz (Münchner Ägyptologische Studien 47).

Westendorf, W. (1962): Grammatik der medizinischen Texte. Berlin (Grundriss der Medizin der alten Ägypter VIII).

Westendorf, W. (1965/1977): Koptisches Handwörterbuch. Heidelberg.

Wilson, P. (1997): A Ptolemaic Lexicon. A Lexicographical Study of the Texts in the Temple of Edfu. Leuven (Orientalia Lovaniensia Analecta 78). 